\title{
La pédagogie comme pivot de l'interdisciplinarité
}

Jacques Hamel

\section{OpenEdition}

Journals

Édition électronique

URL : https://journals.openedition.org/ries/1969

DOI : 10.4000/ries. 1969

ISSN : 2261-4265

\section{Éditeur}

France Education international

\section{Édition imprimée}

Date de publication : 1 septembre 2002

Pagination : 143-151

ISBN : 978-285420-554-1

ISSN : $1254-4590$

\section{Référence électronique}

Jacques Hamel, «La pédagogie comme pivot de l'interdisciplinarité », Revue internationale d'éducation de Sèvres [En ligne], 30 | septembre 2002, mis en ligne le 25 novembre 2011, consulté le 06 juillet 2021. URL : http://journals.openedition.org/ries/1969; DOI : https://doi.org/10.4000/ries.1969

Ce document a été généré automatiquement le 6 juillet 2021

(c) Tous droits réservés 


\title{
La pédagogie comme pivot de l'interdisciplinarité
}

\author{
Jacques Hamel
}

1 Les institutions d'enseignement, en particulier l'université, s'entêtent à vouloir décloisonner les savoirs et à les fusionner en une connaissance capable de rendre compte de la complexité des choses. Les programmes pédagogiques doivent aujourd'hui se plier à ce but et l'enseignement doit être sans retard orienté en conséquence. Les dirigeants du monde de l'éducation lancent des invites en ce sens, parfois sous la forme d'un ordre auquel tout professeur est tenu de se ranger.

2 Or la vogue de l'interdisciplinarité, traduite par une effervescence qui est sans conteste féconde et propice, ne parvient pas à dissimuler son caractère artificieux et les périls qu'elle fait courir à la formation des connaissances et à leur enseignement. Il est donc souhaitable d'envisager de tels écueils afin d'apporter les nuances qui manquent pour que l'interdisciplinarité trouve son droit et sa juste pertinence. La pédagogie joue un rôle primordial à cet égard puisqu'elle se révèle en définitive, comme on tentera de démontrer ici, le fer de lance de cette entreprise destinée à combiner les connaissances nées des spécialités du savoir.

\section{L'interdisciplinarité, pivot des programmes d'enseignement}

3 L'interdisciplinarité est aujourd'hui le terme qui coiffe les divers programmes d'enseignement. L'enseignement primaire et secondaire s'aligne dans une certaine mesure sur cette voie, mais c'est principalement aux niveaux collégial et universitaire qu'on désire battre en brèche la spécialisation des connaissances et cela pour divers motifs.

4 Selon Edgar Morin, l'un de ses plus ardents défenseurs, l'interdisciplinarité s'impose si l'on veut mettre fin au « découpage des disciplines qui rend incapable de saisir "ce qui est tissé ensemble", c'est-à-dire, selon le sens originel du terme, le complexe » (Morin, 
1999a : 14). La division du travail à l'œuvre en science a eu pour effet, soutient-il, «d'apporter les inconvénients de la sur-spécialisation, du cloisonnement et du morcellement du savoir » (Ibid. : 15). La science, en décomposant la réalité, a enrôlé les connaissances nées dans ce sillage à des utilisations techniques hors de tout contrôle. L'interdisciplinarité permet donc de renouer avec le «complexe " qu'a gommé, à tort ou à raison, la science pour remplir sa vocation, et avec la culture humaniste engloutie sous la spécialisation des connaissances exigée par l'application technique.

5 L'interdisciplinarité a pour principe d'envisager la possibilité de «concevoir ce qui relie » les objets entre eux et le développement de l'aptitude à contextualiser et à globaliser les savoirs, cela devenant un «impératif d'éducation » (ibid.: 27). Le but de l'interdisciplinarité n'est donc pas tant « d'ouvrir les frontières entre les disciplines que de transformer ce qui génère ces frontières: les principes organisateurs de la connaissance » (ibid. : 28). L'interdisciplinarité est, dans cette voie, le prélude à un mode d'organisation de la connaissance cher à Morin, la transdisciplinarité, qui fusionnerait instantanément les connaissances en un savoir capable d'embrasser le complexe sur lequel se basent les «entités naturelles sur lesquelles ont toujours porté les grandes interrogations humaines : le cosmos, la vie et, à la limite, l'être humain » (ibid. : 39).

6 S'il paraît facile de donner un visage à l'interdisciplinarité, elle est toutefois compliquée à traduire sous la forme d'un programme d'enseignement. Edgar Morin s'y est risqué en acceptant de présider le Conseil scientifique chargé de faire des suggestions pour l'enseignement des connaissances dans les lycées français (voir Morin, 1999b: 7 et suiv.). L'interdisciplinarité semble chez lui prendre corps par la création de nouvelles divisions entre connaissances portant des noms imagés, marqués au coin de l'humanisme. Il suffit en effet de remplacer sciences naturelles par sciences de la Terre, sciences biologiques par sciences de la vie, sciences humaines par sciences de l'humanité (voir Morin, 1999a: 7-15) et de leur attribuer la volonté de jeter des ponts pour que l'interdisciplinarité trouve son droit et sa légitimité.

Il est difficile de saisir sur cette base les vertus de ces nouvelles dénominations sur des connaissances vers lesquelles elles ouvrent la voie, tout comme du reste sur leur apprentissage. Comment en effet se formule et s'acquiert le savoir qui veut englober le « complexe cosmo-physico-bio-anthropo-social»? L'objet de l'interdisciplinarité et la pédagogie qu'elle sous-entend demeurent des points aveugles de la réforme du savoir proposée qui, d'ailleurs, est restée sans lendemain au grand dam de son auteur pressé d'en découdre avec la science.

\section{L'interdisciplinarité et la science vont-elles de pair?}

8 Car la science est mise au premier chef au banc des accusés pour la raison qu'elle dénature la complexité de la réalité, la transforme en objet inerte et engendre des connaissances spécialisées entre lesquelles il est impossible de jeter des ponts. L'interdisciplinarité vient donc brutalement mettre en cause la science, du moins la conception qu'en donne l'épistémologie. Le philosophe des sciences Gilles-Gaston Granger propose en effet de la définir comme une connaissance par objet et par concepts, elle-même générée par un travail qui obéit à l'objectif de représenter toute chose associée à la "réalité » sous une forme distincte de sa nature propre. Sous ce chef, la science exige le découpage de la réalité en un objet dans le but de la «manipuler » pour en isoler les éléments et tenter de reproduire leur imbrication et 
leur fonctionnement grâce à une représentation expressément et uniquement façonnée par le moyen de symboles et de concepts, c'est-à-dire par des abstractions considérées comme des moyens pour représenter, non pas pour séparer ou disjoindre comme invite à penser ce terme pris au pied de la lettre.

La science ainsi envisagée semble au premier abord prêter flanc aux reproches qui donnent son sens à l'interdisciplinarité. Or, sans vouloir faire preuve d'un scientisme de mauvais aloi, l'anathème jeté sur la science manque singulièrement de nuances. Il convient de les apporter à propos de la façon dont se conçoivent l'objet et le sens épistémologique que revêt la réduction dans son rayon.

En premier lieu, en science, par objet il faut entendre la réduction de la « réalité » que l'une ou l'autre des sciences opère dans le but de traduire sa représentation au moyen de concepts. Cette réduction est nécessaire à la science et en est le premier pas. Sans conteste, réduire revêt un statut positif dans l'orbite de la science. Le terme "réduction" équivaut donc à découper la réalité et à la rendre provisoirement amorphe pour qu'elle s'ouvre de cette manière à la connaissance scientifique que Granger, en mots imagés, conçoit comme un « détour par le royaume infiniment ouvert des abstractions pour rendre plus pénétrant, plus puissant et plus précis le contact avec la réalité » (Granger $1986: 120)$.

11 Le découpage n'a rien ici d'une volonté manifeste de disjoindre et de séparer dans les faits et, par conséquent, de briser définitivement « ce qui est tissé ensemble ». Il obéit à une fonction épistémologique destinée à entrer en contact avec la réalité de façon précise et pénétrante en mettant entre parenthèses, pour ce faire, sa "complexité » puisque c'est là la condition même de la connaissance scientifique.

Dans cette perspective, on voit bien que la complexité, au sens où l'entend Edgar Morin, n'a guère de part ni de droit en science. Il en va de même pour la pensée complexe dont cet auteur se fait l'ardent défenseur. Elle enfreint ou, pour mieux dire, elle outrepasse la visée de la science qui, jusqu'à preuve du contraire, lui donne sa raison d'être en tant que connaissance fondamentale, c'est-à-dire une connaissance basée sur un objet qu'elle cerne et étudie à fond, et dans le cadre d'une discipline dont les adeptes peuvent se targuer d'en être les spécialistes.

Si la science réduit à des fins épistémologiques, c'est-à-dire pour être apte à formuler la connaissance qu'on attend d'elle, engendre-t-elle une attitude réductrice chez ses adeptes ? Il est exagéré de penser que tout scientifique, sur la base de l'objet dont il est spécialiste, s'imagine tout connaître, y compris dans sa propre discipline. Il est plus raisonnable de penser que la spécialisation ne parvient pas à annuler la volonté de savoir. Au contraire, elle l'avive et l'amplifie d'emblée. Le spécialiste, pour justement cibler son objet, doit nécessairement chercher à en cerner les différentes facettes et celles-ci constituent souvent l'objet d'autres spécialités. Les sociologues de l'éducation ne peuvent se permettre d'ignorer les théories et travaux des sociologues de la culture et du langage, ainsi que ceux des psychologues, par exemple. Ils peuvent difficilement faire fi des progrès de la neurobiologie et du domaine des sciences cognitives. La volonté de savoir devient donc une obligation pour qui se réclame d'être un spécialiste. Elle incite tout spécialiste à déborder le rayon des connaissances qui forgent son objet d'élection. 


\section{L'interdisciplinarité et l'application de la connaissance}

14 Comment alors expliquer la soudaine frénésie à vouloir fédérer les disciplines scientifiques et, plus largement, à enrôler par injonction toutes formes de savoir sous l'étendard de l'interdisciplinarité ? Au risque de se voir accusé de manquer de nuance, sinon de prudence, on peut affirmer que l'interdisciplinarité est à l'ordre du jour depuis que des applications pratiques sont attendues de la science, de toutes les sciences, sinon de toute connaissance au nom de la société du savoir proclamée de nos jours à cor et à cris. En effet, la mondialisation de l'économie et l'autoroute de l'information vont à l'encontre de la connaissance qui réduit et isole, et à laquelle on associe abusivement la science, par exemple. Voilà le contexte qui a mis en exergue la " complexité » et la nécessité de " contextualiser et globaliser ». L'interdisciplinarité se fait vertu pour répondre à des problèmes de nature pratique et globale qui, assure-telle, entrave la spécialisation des connaissances scientifiques en vigueur dans leurs lieux de production, comme l'université.

15 Les problèmes écologiques de la planète, la circulation rapide de l'information et des capitaux à l'échelle internationale, l'épidémie du VIH responsable du sida, entre autres, requièrent d'autorité le concert des disciplines scientifiques et l'aptitude à contextualiser et à globaliser que Edgar Morin associe à juste titre à la pensée complexe qu'illustre dans son esprit l'interdisciplinarité.

16 L'application ainsi conçue est entièrement valide et ne saurait être mise en cause. Si le concert des sciences fait partie de la raison d'être de la science, force est d'admettre que c'est bel et bien ce contexte qui l'a fait aboutir. Il est responsable de la nécessité de jeter des ponts entre sciences, de coordonner les connaissances qu'elles produisent, de s'ouvrir à la pensée philosophique et pratique.

17 Toutefois l'application qui rend justice à l'interdisciplinarité soulève des questions. Sans en renier la nécessité, doit-elle être la raison voire l'unique motif qui commande aujourd'hui la production des connaissances scientifiques et autres? Faut-il admettre que tout savoir doit être d'office canalisé vers l'application? Que l'université, sinon l'école, soit orientée dans sa direction, point final? Que des injonctions de tous ordres sont lancées afin que toute connaissance se plie immédiatement à la résolution de problèmes pratiques? La spécialisation doit-elle conserver son droit d'exister à l'université, par exemple? L'interdisciplinarité, alignée strictement sur l'application, risque de faire préjudice à la science, certes, mais également à la nécessaire spécialisation des autres domaines du savoir : humanités, philosophie et art.

Résoudre des problèmes sur le plan pratique commande en effet une action et celle-ci rappelle que la société, par exemple, est en réalité complexe et marquée par l'interdépendance de ce qui la constitue. Elle oblige ainsi à la levée des réductions opérées par les disciplines scientifiques, telle la sociologie, dans le but d'en obtenir un contact «plus pénétrant, plus puissant, plus précis» sous forme d'un objet d'étude. C'est bel et bien dans cette perspective que les disciplines et leurs spécialités trahissent leurs limites; or celles-ci ne portent aucun préjudice aux réductions nécessaires à leur vocation première. L'annulation de ces réductions engendre alors un contact qui ne relève plus d'une visée de connaissance, mais d'une action. L'application devient alors nécessité et le dialogue entre disciplines scientifiques indispensable. 

ratés. Le dialogue entre disciplines est loin d'être facile à établir dans ce but. Le fonctionnement chaotique et la pauvreté des résultats de recherche des équipes interdisciplinaires en témoignent sans conteste. Le concert des disciplines scientifiques exige la levée, voulue et provisoire, des objets et des théories spécialisés. Il oblige du même coup à traduire les connaissances acquises dans chaque domaine dans la veine des autres disciplines qui entrent en ligne de compte. Il apparaît vite que les spécialistes des disciplines sont, pour remplir cet office, de piètres candidats. L'interdisciplinarité réclame les services de traduction ou de médiation apparentés à des enjeux intellectuels qui débordent largement la science. À mon sens, ils sont d'ordre philosophique. En effet, ils ont trait au sens que revêt la connaissance, toute connaissance, considérée comme expérience de l'espèce humaine alignée sur sa volonté de savoir et sa survie biologique. Sans vouloir insister sur ce point, il appert que l'interdisciplinarité relève en définitive de la philosophie de la connaissance propre à "décrire et à faire comprendre le sens, la portée et les procédures de l'effort de rationalisation qu'exprime le mouvement de la science » (Granger, 1986: 114) et celui des autres formes de la connaissance.

La philosophie se démarque de la science par le fait qu'elle n'est pas une connaissance par objet, née de la réduction de la réalité, mais une démarche interprétative capable d'éclairer le sens d'une entreprise comme la science où dominent les savoirs spécialisés. Sous son égide et dans cette optique, le dialogue entre sciences peut être harmonieusement créé tout comme, du reste, peuvent s'établir des liens sur la lancée entre la science et la philosophie, l'art, voire la religion. L'interdisciplinarité révèle ainsi sa véritable nature, philosophique.

\section{Survol rapide des expériences de programmes d'enseignement sous le signe de l'interdisciplinarité}

L'enseignement doit être également en harmonie avec l'interdisciplinarité afin de battre en brèche la spécialisation à l'honneur depuis la réforme de l'université proposée par Humboldt en 1809 et au nom de laquelle fleurissent les départements propres à chaque science et, de ce fait, à chaque spécialisation du savoir. La culture scientifique s'est, depuis, écartée des humanités, de la philosophie et de l'art pour en venir, par son formidable développement, à les placer sous son joug. Il faut donc inventer des programmes éducatifs capables de relier les connaissances spécialisées dans le but de donner une vision globale à l'image de la phrase de Pascal: " La connaissance $\mathrm{du}$ tout a besoin de celle des parties qui ont besoin de celle du tout. » La pensée du philosophe trouverait acte dans les leçons de connexion grâce auxquelles, selon Edgar Morin, on enseignerait que « l'homme est à la fois totalement biologique et totalement culturel, que le cerveau étudié en biologie et l'esprit étudié en psychologie sont les deux faces d'une même réalité, et soulignant que l'émergence de l'esprit suppose le langage et la culture » (Morin, 1999a : 88).

Souscrire à cette orientation sous-tend dans les foyers d'éducation « le recours, non pas à des enseignements séparés, mais à une pédagogie conjointe groupant philosophe, psychologue, sociologue, historien, écrivain et ceci se conjuguerait avec une initiation à la lucidité » (Morin, 1999a : 56). La formule et les moyens pour parvenir à cette fin restent toutefois les points aveugles de cette pédagogie ouverte dans son principe à

Revue internationale d'éducation de Sèvres, 30 | septembre 2002 
l'interdisciplinarité. Des expériences ont été tentées dans ce sens et servent de laboratoire d'initiation à la lucidité.

Voilà près de trente ans, l'université de Montréal a résolu de créer la faculté des Arts et des Sciences dans le but de nouer des liens entre les sciences, les sciences sociales, les humanités et les arts. L'entreprise se réclamait des universités américaines où, sous l'égide des Arts and Sciences, les étudiants sont conviés à se frotter aux sciences et à la philosophie, à la littérature et aux humanités, dans l'espoir d'en faire des esprits meublés par un ensemble de connaissances dignes de ce nom.

À l'initiative de la dite faculté, les programmes des diverses disciplines ont d'abord incité, puis contraint les étudiants à s'inscrire à des blocs de cours d'autres disciplines, sans grand succès. Ce choix demeurant libre, aucun étudiant ne le faisait de son plein gré. L'obligation qui leur est désormais faite en ce sens conduit les étudiants à suivre ces cours durant la dernière session de leur baccalauréat, session d'ailleurs considérée par eux comme une épreuve propre à diminuer la moyenne cumulative de leurs notes.

L'évaluation de cette formule destinée à orienter l'enseignement vers l'interdisciplinarité montre que les étudiants sont loin de se familiariser avec la « complexité » et l'" aptitude à globaliser et contextualiser ». L'incursion dans un autre domaine du savoir à laquelle ils ont été contraints leur a prouvé noir sur blanc qu'ils sont les spécialistes d'une discipline et que, pour cette raison, en maîtriser une autre se révèle une rude épreuve. Les leçons de philosophie ont certes leur intérêt pour les étudiants de sociologie qui, toutefois, ne tardent pas à découvrir les limites de leur culture philosophique et de leur motivation à l'acquérir qui se cantonne à vouloir donner du relief à leur discipline d'élection, la sociologie.

Ces étudiants sont, d'autre part, nombreux à n'avoir pas spécialement goûté l'enseignement de la philosophie, entre autres parce que la pédagogie mise de l'avant n'était pas particulièrement adaptée à un auditoire de néophytes.

\section{La pédagogie comme vecteur d'interdisciplinarité}

27 Car l'interdisciplinarité n'est pas sans incidence sur la pédagogie. Elle soulève des enjeux de cet ordre qu'on aurait tort de ne pas envisager. Tout professeur titulaire de cours expressément destinés à un large public, toutes disciplines confondues, peut les mesurer sur le vif, obligé qu'il est de reconnaître les limites de sa culture interdisciplinaire et d'admettre que les ponts qu'il jette entre diverses disciplines s'élaborent en fonction de leur supposée contribution à sa propre discipline.

Sans ce point d'attache, la pédagogie placée sous l'autorité envahissante de l'interdisciplinarité peut rapidement conduire, à mon sens, à la dilution des contenus de cours. La vogue de l'interdisciplinarité masque donc des débats qu'il faudrait avoir le courage d'engager dans l'intention d'établir des liens entre disciplines. L'invitation à s'initier à diverses disciplines s'alignera sur des buts et des motifs qui auront l'éclat et la légitimité de la formation diversifiée à laquelle on associe l'interdisciplinarité. Il restera néanmoins à traduire cette formation diversifiée en une démarche d'apprentissage qui n'ait pas l'allure de la mosaïque ou de l'éparpillement.

Nombre de professeurs s'ingénient à concevoir les formules pédagogiques indispensables au dialogue fécond entre sciences, entre connaissance scientifique et connaissance philosophique, entre celle-ci et la pensée qu'engendrent l'art, la politique, 
le journalisme et le cyberespace. Le bricolage pédagogique auquel ils s'adonnent, par essais et erreurs, revêt une richesse souvent ignorée ou passée sous silence dans les écrits sur l'interdisciplinarité. Or, me semble-t-il, il témoigne de la philosophie de la connaissance à laquelle correspond dans mon esprit l'interdisciplinarité et en constitue la clef de voûte.

30 L'interdisciplinarité s'affiche dans cette perspective comme une philosophie de l'éducation dont il faudrait circonscrire le visage en exhibant et en systématisant le bricolage dont font foi les formules pédagogiques mises au point dans le but de faire communiquer les connaissances. Elles lui donnent acte et en constituent le terrain d'exercice par excellence. Voilà pourquoi il faudrait sonder les initiatives et formules pédagogiques surgies de l'esprit des pédagogues pour fondre des connaissances au nom d'un problème pratique auquel on veut remédier, ou d'un thème qu'on refuse d'envisager en tant qu'objet propre à la science ou à l'une ou l'autre de ses disciplines. Sur cette base, il importe de mettre à jour les ressorts de la pédagogie pour découvrir les lignes directrices de la pensée en raison de laquelle prend corps le dialogue des connaissances. Le vocabulaire utilisé, les passerelles qu'il jette et les exemples auxquels on recourt pour l'illustrer devraient être passés au crible afin de découvrir à l'œuvre dans la pédagogie la traduction ou la médiation sur laquelle s'appuie l'interdisciplinarité.

31 Sur la lancée - l'expression le trahissant - l'interdisciplinarité s'aligne en définitive sur le «bricolage intellectuel» évoqué par Claude Lévi-Strauss pour qualifier la pensée mythique comme la forme de pensée qui recourt principalement au raisonnement analogique et qui construit sur cette base des ensembles ordonnés en mobilisant toutes les homologies repérables dans les divers domaines de l'expérience sensible. Les formules pédagogiques en sont l'antichambre, sinon la porte d'entrée.

Les professeurs chargés d'établir des liens entre des connaissances de divers ordres orchestrent à cette fin une pédagogie qui recèle en elle la formule grâce à laquelle l'interdisciplinarité trouve acte. En effet, à coups d'analogie et de rapprochements, ils parviennent à introduire un fil d'Ariane entre des connaissances sans rapport évident, pour ainsi les gratifier de la capacité d'éclairer la «complexité » en recomposant la « réalité » sous sa forme de totalité et, dans cette voie, être en mesure de répondre aux " grandes questions humaines : le cosmos, la vie et l'être humain ». Ils puisent dans leur répertoire de connaissances, plus ou moins étendu, et, avec imagination, par dosage relatif, les enchaînent en outrepassant la visée, le découpage et l'objet que renferme chacune d'entre elles. Les professeurs doivent être aptes à formuler le plus large savoir possible sans le subordonner aux objets et aux concepts des spécialistes.

La pédagogie n'est donc pas sans analogie avec le bricolage sur le plan pratique. Le bricoleur est en effet « apte à exécuter un grand nombre de tâches diversifiées ; mais, à la différence de l'ingénieur, il ne subordonne pas chacune d'elles à l'obtention de matières premières et d'outils conçus et procurés à la mesure de son projet " (LéviStrauss, $1962: 31$ ). Elle correspond dans cette voie au bricolage intellectuel à l'œuvre dans la pensée mythique que cible le chef de l'anthropologie structurale. Selon LéviStrauss, la réflexion mythique se situe à mi-chemin entre "les images concrètes et les concepts » et, de ce fait, fait office d'intermédiaire que décrit éloquemment l'exemple du bricoleur. Si on le regarde à l'œuvre, « excité par son projet, sa première démarche pratique est pourtant rétrospective: il doit se retourner vers un ensemble déjà constitué, formé d'outils et de matériaux; en faire, ou en refaire, l'inventaire ; enfin et 
surtout, engager avec lui une sorte de dialogue, pour répertorier, avant de choisir entre elles, les réponses possibles que l'ensemble peut offrir au problème qu'il lui pose " (ibid. : 32).

Il ne saurait y avoir meilleure illustration de la pédagogie ouverte dans son principe à l'interdisciplinarité. Celle-ci, on l'a vu, revêt une nature philosophique et correspond en définitive à une philosophie de l'éducation propre à percer à jour les formules pédagogiques en vertu desquelles s'enchaînent les connaissances. À l'instar de la philosophie de la connaissance, elle doit chercher à décrire et à comprendre le sens, la portée et les procédures qui donnent corps à l'interdisciplinarité en ciblant la pédagogie qui en est le principal vecteur.

\section{BIBLIOGRAPHIE}

GRANGER Gilles-Gaston (1986) : «Pour une épistémologie du travail scientifique », dans Jean Hamburger (dir.), La philosophie des sciences aujourd'hui. Paris : Gauthier-Villars : 111-122.

LÉVI-STRAUSS Claude (1962) : La pensée sauvage. Paris : Plon.

MORIN Edgar (1999a) : La tête bien faite. Repenser la réforme, réformer la pensée. Paris : Seuil.

MORIN Edgar (1999b) : «Introduction », dans Relier les connaissances. Paris : Seuil : 7-15.

MORIN Edgar (2000) : Les sept savoirs nécessaires à l'éducation du futur. Paris : Seuil.

\section{RÉSUMÉS}

L'interdisciplinarité jouit d'une période de gloire. L'entreprise qui consiste à vouloir conjuguer diverses connaissances spécialisées a le vent en poupe. Mise en avant dans les différentes institutions de recherche et d'enseignement, elle semble promise au succès. L'interdisciplinarité est la formule qui permettrait de sortir de l'impasse de la spécialisation du savoir amenée par le développement de la science; or cette spécialisation fait obstacle aujourd'hui à la formation de connaissances propres à établir des ponts entre les objets, les domaines et les aspects de la « réalité » qu'elle s'évertue à réduire et à isoler sous couleur d'expliquer exactement les choses. L'interdisciplinarité se voit donc prônée dans le feu du procès intenté à la science ainsi qu'aux connaissances dites spécialisées, étendues à celles qui sont générées par la philosophie, les humanités et l'art.

\section{INDEX}

Mots-clés : interdisciplinarité, sciences de l'éducation, pédagogie 


\section{AUTEUR}

\section{JACQUES HAMEL}

Professeur titulaire au département de sociologie de l'Université de Montréal et professeur associé à l'Observatoire Jeunes et Société (Institution nationale de la recherche scientifique) [hamelja@SOcio.UMontreal.CA] 